\title{
Transcatheter closure for the treatment of pseudoventricular aneurysm after acute myocardial infarction: a case report
}

\author{
Wei Zhong ${ }^{1,2,3}$, Zhidong Liu ${ }^{1,2,3}$, Weixiong Fan $^{1,2,3,4}$, Bin Ou ${ }^{1,2,3}$, Min Zhong ${ }^{1,2,3}$, Zhiwen Zeng ${ }^{1,2,3}$, \\ Xianfang Wang ${ }^{1,2,3}$, Wilbert S. Aronow ${ }^{5}$, Francesco Nappi ${ }^{6}$, Juan Lacalzada-Almeida ${ }^{7}$, Zhixiong Zhong ${ }^{1,2,3}$ \\ ${ }^{1}$ Center for Cardiovascular Diseases, Meizhou People's Hospital (Huangtang Hospital), Meizhou Hospital Affiliated to Sun Yat-sen University, \\ Meizhou, China; ${ }^{2}$ Guangdong Provincial Engineering and Technology Research Center for Molecular Diagnostics of Cardiovascular Diseases, \\ Meizhou, China; ${ }^{3}$ Guangdong Provincial Key Laboratory of Precision Medicine and Clinical Translational Research of Hakka Population, Meizhou, \\ China; ${ }^{4}$ Magnetic Resonance Department, Meizhou People's Hospital (Huangtang Hospital), Meizhou Hospital Affiliated to Sun Yat-sen University, \\ Meizhou, China; ${ }^{5}$ Cardiology Department, Westchester Medical Center and New York Medical College, Valhalla, NY, USA; ${ }^{6}$ Department of Cardiac \\ Surgery, Centre Cardiologique du Nord de Saint-Denis, Paris, France; ${ }^{7}$ Cardiac Imaging Laboratory, Department of Cardiology, University Hospital \\ of Canary Islands, La Cuesta, La Laguna, Tenerife, Spain \\ Correspondence to: Zhixiong Zhong. Center for Cardiovascular Diseases, Meizhou People's Hospital (Huangtang Hospital), Meizhou Hospital \\ Affiliated to Sun Yat-sen University, No. 63 Huangtang Road, Meijiang District, Meizhou 514031, China. Email: zhongzx@aliyun.com.
}

\begin{abstract}
Left ventricular free wall rupture (LVFWR) is a rare but lethal complication of acute myocardial infarction (AMI). Urgent surgery is essential but associated with high postoperative mortality. Even worse, LVFWR patients may experience sudden death without a chance for surgery. In this article, we report our successful transcatheter closure of a patient with the most extensive pseudoventricular aneurysm after AMI reported thus far. Cardiac magnetic resonance imaging (MRI) revealed a giant pseudoventricular aneurysm located in the inferior and lateral walls of the left ventricle; the rupture diameter was $28 \mathrm{~mm}$, and the maximum tumor diameter was $90.2 \mathrm{~mm}$. We used transcarotid approach (TCA) and atrial septal defect closure umbrella to complete the operation, which solved the lack of special interventional instruments to treat pseudoventricular aneurysm after AMI. In addition, we still needed to treat liver and kidney failure caused by hemolysis after operation, and undergone strict follow-up. In conclusion, transcatheter closure is practical and feasible for the treatment of pseudoventricular aneurysm after AMI, although hemolysis and decline of cardiac pumping function after the successful interventional treatment deserve special attention. Future multicenter studies are required to identify patients best suited for interventional treatment timing. And further developments in devices and delivery techniques are required in order to optimize interventional outcomes.
\end{abstract}

Keywords: Transcatheterclosure; acute myocardial infarction (AMI); pseudoventricular aneurysm

Submitted Sep 09, 2020. Accepted for publication Oct 24, 2020.

doi: 10.21037/atm-20-6335

View this article at: http://dx.doi.org/10.21037/atm-20-6335

\section{Introduction}

Left ventricular free wall rupture (LVFWR) occurs in about $2-4 \%$ of all patients with acute myocardial infarction (AMI) and may be accompanied by an unpredictable risk of sudden cardiac death (1-3). Most patients have a very short interval from disease onset to death, with little chance for treatment. In only a small number of patients with pseudoventricular aneurysm, the blood that leaks out of the cardiac cavity is fortunately wrapped, which permits the opportunity for surgery. Traditionally, LVFWR is treated by surgery. However, the postoperative mortality rate is up to $10 \%$, making LVFWR a "forbidden zone" for surgical treatment (4). In recent years, with the advances in interventional technology, transcatheter closure of LVFWR have been reported in several cases (5-8). To our knowledge, 

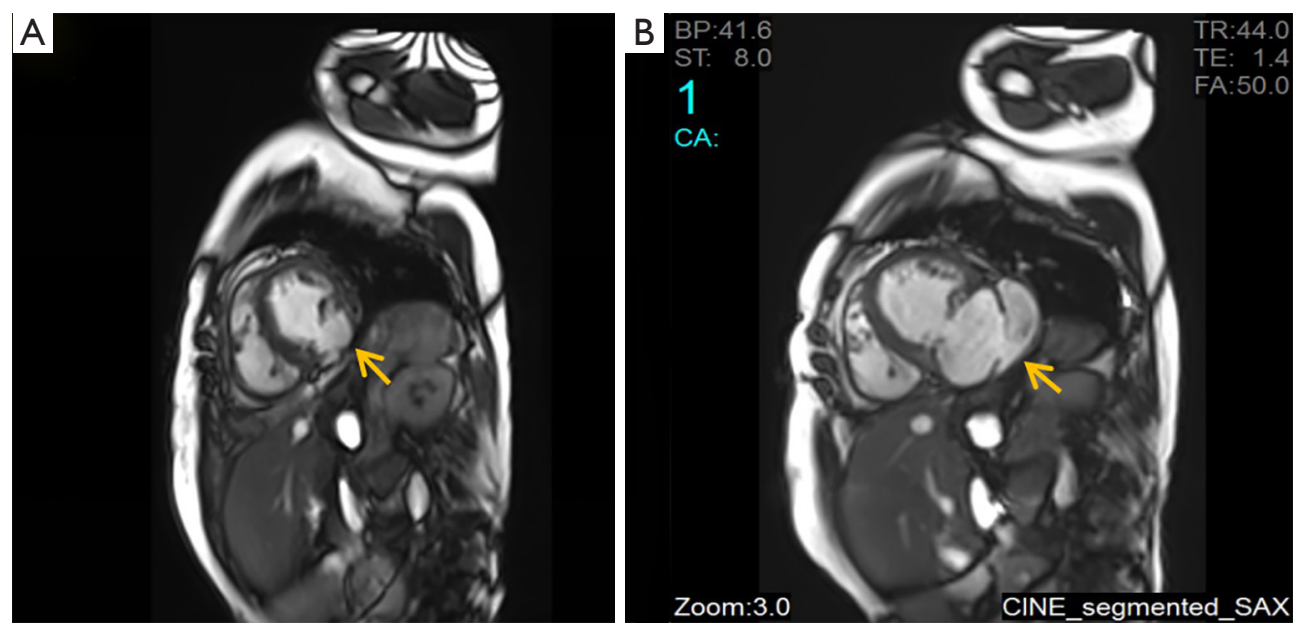

Figure 1 Cardiac MRI images: (A) LGE-CMR finding of the pseudoventricular aneurysm on May 10 (yellow arrow: pseudoventricular aneurysm $40.9 \mathrm{~mm}$ ); (B) LGE-CMR from May 31 reveals the rapid increase of the diameters of the rupture in the left ventricle and the pseudoventricular aneurysm (yellow arrow: pseudoventricular aneurysm $90.2 \mathrm{~mm}$ ). MRI, magnetic resonance imaging; LGE-CMR, late gadolinium-enhanced cardiac magnetic resonance.

the patient described in our current article had the most extensive pseudoventricular aneurysm reported, which was successfully closed in our center.

We present the following case in accordance with the CARE reporting checklist (available at http://dx.doi. org/10.21037/atm-20-6335).

\section{Case presentation}

A 66-year-old woman was admitted to our center on June 2, 2018 with a confirmed diagnosis of AMI. She had undergone mitral valve replacement with St. Jude Medical E100-29M biological valve in 2012 due to mitral valve prolapse syndrome and severe mitral incompetence. Coronary angiography performed on the second day after her admission showed that the distal left circumflex coronary artery was completely occluded. Since the vessel was tine, no stent was implanted. Echocardiography revealed the presence of LVFWR and pseudoventricular aneurysm. Cardiac magnetic resonance imaging (MRI) performed on May 10 and May 31, 2018, revealed a giant pseudoventricular aneurysm located in the inferior and lateral walls of the left ventricle; the rupture diameter increased from 22 to $28 \mathrm{~mm}$, and the maximum tumor diameter increased from 40.9 to $90.2 \mathrm{~mm}$ (Figure 1A,B). The patient developed discomforts such as orthopnea and anorexia, along with worsening symptoms. Blood biochemical tests showed her NT-proBNP was
$>35,000 \mathrm{pg} / \mathrm{mL}$. Since the patient had become critically ill and her heart rupture had lasted 4 weeks, transcatheter closure under general anesthesia was performed on June 5 . An incision was made to expose the left carotid artery (CA), and left heart ventricular angiography performed through the CA revealed the presence of a giant pseudoventricular aneurysm. The diameter of the rupture at the ventricle was about $30 \mathrm{~mm}$. A CA-ascending aorta-left ventriclepseudoventricular aneurysm track was established, and a 36-mm atrial septal occlusion device (SHSMA, Shanghai, China) was successfully placed. Left ventricular (LV) angiography and cardiac ultrasound were performed immediately after operation. It was found that the occlusion device was fixed to the LVFWR, and there was only a small number of residual shunts in the pseudoventricular aneurysm (Figure 2A,B).

Biochemistry performed after the patient returned to the ICU showed the following: NT-proBNP, $>35,000 \mathrm{pg} / \mathrm{mL}$; aspartate aminotransferase (AST), 6,061 U/L; alanine aminotransferase (ALT), 3,278 U/L; creatinine (Cr), $244 \mu \mathrm{mol} / \mathrm{L}$; hemoglobin $(\mathrm{Hgb}), 85 \mathrm{~g} / \mathrm{L}$; and platelet (PLT), 30×10\% L. Red blood cell (RBC) morphology showed different $\mathrm{RBC}$ shapes, along with visible $\mathrm{RBC}$ fragments, suggesting the presence of hemolysis and the accompanying cardiac, hepatic, and renal failure. After treatments including intensified oral rehydration therapy (ORT), alkalization of urine, hormone shock, cardiotonic, diuretics, and plasma exchange, blood biochemistry showed 

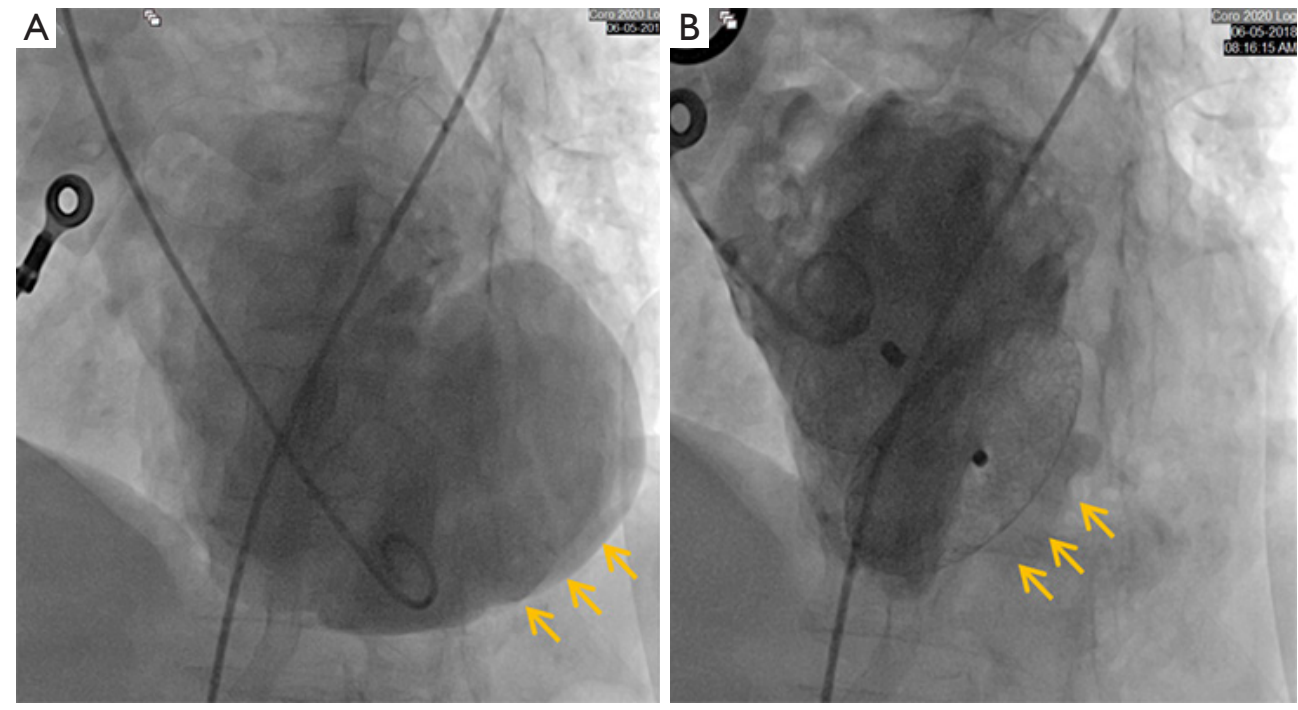

Figure 2 The immediate effect of occlusion: (A) a giant pseudoventricular aneurysm is visible on the left ventricle angiogram before closure (yellow arrow: a giant pseudoventricular aneurysm); (B) LV angiography reveals a small number of shunts inside the pseudoventricular aneurysm after closure (yellow arrow: giant pseudoventricular aneurysm disappeared and a small number of shunts). LV, left ventricular.

the disease condition was improved: NT-proBNP, $1,861 \mathrm{pg} / \mathrm{mL}$; AST, $58 \mathrm{U} / \mathrm{L}$; ALT, $27 \mathrm{U} / \mathrm{L}$; Cr, 120 mol/L; Hgb, $95 \mathrm{~g} / \mathrm{L}$; and PLT, $133 \times 10^{9} / \mathrm{L}$. The New York Heart Association (NYHA) cardiac function was improved from class IV to class II, the hepatic and renal functions and PLTs returned to normal, and Hgb level increased. The patient was then discharged home. The patient did not receive regular follow-up. The telephone visit revealed that she died 2 months after discharge.

All procedures performed in studies involving human participants were in accordance with the ethical standards of the institutional and national research committees, and with the Helsinki Declaration (as revised in 2013). Written informed consent was obtained from the patient for publication of this manuscript and any accompanying images.

\section{Discussion}

Treatment of LVFWR after AMI is complicated. Surgery is the traditional treatment for LVFWR, but only those patients with the formation of pseudoventricular aneurysm may have a chance for surgery, and it is a "forbidden zone" for cardiac surgeons due to the high postoperative mortality rate. In recent years, the advances in interventional technology have shed new light on the treatment of LVFWR. However, due to the different morphologies and positions of LVFWR after AMI and the limitations of surgical instruments, the development of this technique still faces many challenges. An experienced surgeon may consider transcatheter closure of a LV pseudoventricular aneurysm in advance if there is rapid progression of the pseudoventricular aneurysm.

Regarding the procedure, the transcatheter closure of LVFWR is a mechanical intervention in which an atrial septal occlusion device is used to treat a pseudoaneurysm of the left ventricle developing as a complication of AMI. The rupture of the LV free wall is one of the main mechanical complications that occur after AMI with two other catastrophic sequelae; rupture of the ventricular septum and the acute mitral valve regurgitation. The procedure that uses transcarotid approach (TCA) avoids a median sternotomy; however, this is a relatively new concept for which further iterative learning may be needed, unlike the effectiveness of the standard surgical procedure. Besides, TCA can be performed without cardiopulmonary bypass; however, this strategy involves a significant skillset from the interventional cardiologist to operate on the beating heart to allow the elusive perfect adherence of the device which is often more difficult. The recent use of sutureless repair for postinfarction LVFWR has been described. In this approach, patients undergo a minimally invasive repair of the $\mathrm{LV}$ wall with the use of the TachoComb (CSL Behring, Tokyo, Japan) or TachoSil (Nycomed, Zurich, Switzerland) patches. Using this approach, no patients required cardiopulmonary 
bypass. Results showed that recipients of sutureless repair who had blow-out type LVFWR experienced re-rupture. The strategy using TachoComb/TachoSil patches may be a viable treatment option for LVFWR; however, meticulous care should be taken when applying this technique in cases of the blow-out type LVFWR (9). Further investigations are required to establish whether the TCA approach or the use of a sutureless procedure provides an ongoing benefit over the traditional open repair of the LVFWR with or without the development of a pseudoaneurysm in terms of low morbidity and mortality.

Nappi et al. (10) described the presence of a LVFWR associated with ischemic mitral valve prolapse, or the latter in combination with an interventricular septal rupture. A combination of any two types of rupture is rare and coined "ventricular double rupture" (VDR). This pathoanatomic condition demonstrates a specific clinical course that is independent of $\mathrm{LV}$ chamber size and ventricular function before the calamitous event. It is important to note that none of the cases of rupture of the LVFWR with or without ischemic mitral valve prolapse showed evidence of $\mathrm{LV}$ dilatation or evidence of heart failure prior to AMI complicated by rupture (10). Tanaka et al. (11) described 10 patients with VDR suggesting that the ventricular septal rupture in the apical region is a precursor of VDR and requires immediate surgery. Infrequently, the ruptured LV free wall can be encompassed by the pericardium, constituting a pseudoaneurysm $(10,12)$. Nappi et al. noted that the ischemic mitral prolapse with or without heart failure seems to predetermine a high percentage of deaths during the early phase of AMI. The rupture of the ventricular wall occurs as a sudden event with cataclysmic clinical signs or and a dire course. As also described by other authors, Nappi et al. highlighted that the rupture of the free wall of the left ventricle commonly happens in patients with 'fatty' hearts and those without evidence of previous heart failure $(10,13)$.

It should be noted that in our case 6 years later the patient was hospitalized for the development of an extensive pseudoaneurysm of her left ventricle secondary to an AMI with total occlusion of the distal left circumflex coronary artery. Nappi et al. remarked that although the primary lesion of the papillary muscle (PM) was responsible for leaflet prolapse with a rate of $66.7 \%$ for the posteromedial papillary muscle (PMPM) and 20\% for the anterolateral; however in a fraction of cases, the ischemic mitral prolapse may be associated to the necrosis of a restricted area of the myocardium adjacent to the PM, resulting in abnormal traction and subsequent dyssynchrony $(11,14-16)$. We could hypothesize that the substrate of mitral disease that has been treated with a mechanical prosthesis has been intertwined with an evolutionary ischemic component. In fact, the pseudoaneurysm developed in the lateral and inferior region of the myocardium corresponding to the scar tissue of the myocardial infarction (MI), which has vasculature dependent on the circumflex artery. We observed that ischemic traction primarily affects the PMPM which is vascularized by the right coronary artery or circumflex artery (10).

To our knowledge, our current patient had the largest diameter $(30 \mathrm{~mm})$ of the rupture in the left ventricle, and the largest diameter $(90.2 \mathrm{~mm})$ of pseudoventricular aneurysm reported thus far, and no such case has been successfully treated. At present, the largest waist diameter of the septal occluder is only $28 \mathrm{~mm}$ in China. As the choice of occlusion devices was highly limited, we chose a $36-\mathrm{mm}$ septal occluder before the operation. The timing of the operation was decided after 4 weeks. Cardiac MRI revealed that the diameters of the rupture and the pseudoventricular aneurysm were continually increasing. The operation was performed 4 weeks after the pseudoventricular aneurysm was found, which met the recommendations of the current expert consensus $(17,18)$. Besides, the operation was performed via a TCA, which was different from the conventional femoral approach and based on the following considerations: (I) the length of the available delivery sheath at the time was only $80 \mathrm{~cm}$, and it was difficult to reach the pseudoventricular aneurysm from the femoral artery; (II) the TCA is shorter and straighter than the femoral approach and can obviate the risk of sudden rupture of the tumor caused by the high tension of the guidewire during the operation. The operative procedure was successfully conducted in full accordance with the plan mentioned above.

Hemolysis is caused by blood being forced through a small opening at high pressure. Hemolysis occurred after transcatheter closure, mainly due to the use of the septal occluder. The high-pressure gradient between the left and right ventricles and the fewer polyester patches and thinner wire weaving in the septal occluder resulted in the lysis of a large number of RBCs under the impact of high-speed blood flow. After active treatment, the patient was discharged after her condition improved. Unfortunately, a telephone visit revealed that she died 2 months after discharge. In addition to the irregular follow-up, the main cause of her death was that the considerable LVFWR resulted in the loss of a large amount of normal myocardial tissue in the heart, leading to the inevitable decline in cardiac pumping function, resulting in sudden cardiac death. 
In summary, transcatheter closure is practical and feasible for the treatment of pseudoventricular aneurysm after AMI, although hemolysis and decline of cardiac pumping function after the successful interventional treatment deserve special attention.

\section{Several questions to be further discussed}

Question 1. For patients with LV pseudoventricular aneurysm progress rapidly, can transcatbeter closure be performed in advance?

\section{Expert opinion 1: Dr. Wilbert S. Aronow}

Yes, transcatheter closure be performed in advance for patients with $L V$ pseudoventricular aneurysm progress rapidly.

\section{Expert opinion 2: Dr. Juan Lacalzada-Almeida}

Most LV pseudoaneurysms in patients who experience a $\mathrm{MI}$ and reach the cardiac surgery operating room are small, probably because larger LV pseudoaneurysms rupture and never reach the operating room. The high risk of death because of rupture and cardiac tamponade associated with $\mathrm{LV}$ pseudoaneurysm is well known. In the case presented, mortality associated with medical treatment was higher than that associated with surgical treatment (approximately $48 \%$ vs. $20 \%$, respectively). Therefore, we think that the approach taken by the authors was correct, since the diameter of the rupture grew, as did that of the aneurysm. The percutaneous procedure was preferable to surgery, which carries a high risk, especially because the patient had previously undergone mitral valve surgery and had a totally occluded distal left circumflex coronary artery that was not going to be revascularized. In addition, the presence of other comorbidities warrants performance of a percutaneous procedure. Moreover, surgery to treat large LV pseudoaneurysm would require wide resection of neighboring healthy myocardial tissue and would therefore diminish the pumping function of the heart during the postoperative period. Percutaneous closure of a ruptured LV free wall after MI has been reported as a bridge to definitive surgical management.

\section{Expert opinion 3: Dr. Francesco Nappi}

Recently, there has been a new interest in the CA access for percutaneous intervention during the transcatheter valve therapy (TVT) procedure (19-21). Among surgeons, the use of this approach has established the role of the
CA as an alternative to the trans-apical approach, giving surgeons the opportunity to perform transcatheter aortic valve replacement (TAVR) procedures with/without the collaboration of the cardiologists. Among cardiologists, the TCA has proven to be a viable alternative to the classic femoral approach for percutaneous TVT interventions (11-13); thus TCA procedures, which utilizes collaboration between surgeons and cardiologists have been very beneficial and is becoming increasingly popular.

This convergence of interests, however, may raise concerns that TCA could be used extensively as an alternative to surgery in managing AMI complications, predisposing to the onset of complications that mitigate the short- and long-term survival benefits offered by traditional surgery. To date, there are no guidelines for the approach to CA in patients in whom TAVR is recommended or for the management of AMI complications (22).

In this case report, the authors provide the results of the experience with the use of the TCA approach for the percutaneous closure of a LVFWR which caused the formation of a ventricular pseudoaneurysm. They suggest a discussion of the optimal use of the TCA approach in patients with AMI complications.

Zhong et al. used a $36-\mathrm{mm}$ atrial septal occlusion device (SHSMA, Shanghai, China) to close the LVFWR and treat the giant pseudoaneurysm. The procedure was performed 4 weeks after hospitalization, although it would have been more useful to perform it within 48 hours. As for the choice of the TCA, it was highly effective. The use of the transfemoral approach was not favorable because it was limited to $80 \mathrm{~cm}$ of the available delivery sheath thus making it difficult to reach the pseudoaneurysm from the femoral artery. Conversely, the distance between the TCA and the lesion to be treated is shorter. Furthermore, in these conditions, handling the material used for the procedure in closer proximity to the pseudoaneurysm avoids the risk of iatrogenic injury caused by the high tension exerted by the guidewire during the operation.

Delaying the intervention may cause the progressive deterioration of ventricular function with an extension of the lesion thereby limiting the effectiveness of the device that is used.

The left ventricle has the configuration of a cylinder. Ventricular mechanics during systole involves moving the lower base of the left ventricle towards the upper base while simultaneously time rotating the ventricular chamber which pivots on the interventricular septum (23). The mechanical 
stress to which the ventricular muscle is subjected is very high and results in an unfavorable effect both on the primary lesion and on the device that has been used.

We note that two issues may occur as a result of this:

(I) The rupture of the ventricular wall tends progressively to increase in the diameter;

(II) The attachment surface of the device can be irregular thus limiting its effectiveness.

\section{Question 2. How can bemolytic reactions be prevented after occlusion in such patients?}

\section{Expert opinion 1: Dr. Wilbert S. Aronow}

Hemolysis is caused by blood being forced through a small opening at high pressure. There must have been a small hole in the occlusion device causing the hemolysis. The only way to prevent hemolysis is to make sure that there is no small hole in the occlusion device.

\section{Expert opinion 2: Dr. Juan Lacalzada-Almeida}

Various authors have reported the potential complication of hemolytic anemia by turbulent flow and the cut-off force of occlusion devices in this type of patient. Turbulent flow entering and exiting between the LV pseudoaneurysm and the LV cavity and detected using color flow Doppler could be produced, as in the case reported, by residual shunts in the area of the closure of the occlusion device of the $\mathrm{LV}$ pseudoaneurysm. There have been reports of cases of severe hemolysis, hyperbilirubinemia, and kidney failure after percutaneous closure of the $\mathrm{LV}$ pseudoaneurysm with an Amplatzer septal occluder (ASO), together with multiple residual shunts between the $\mathrm{LV}$ pseudoaneurysm and the $\mathrm{LV}$ cavity, although this was resolved after conversion to conventional surgery. In the present case, the problem probably arises because when such large devices are used, they can become trapped by the mesh around the border of the ventricular defect, thus preventing complete sealing and leading to multiple residual shunts and subsequent hemolysis. The paucity of reports on hemolysis after implantation of an ASO for closure of $\mathrm{LV}$ pseudoaneurysm reflects the limited number of cases in this field. In the present case, spontaneous sealing of the residual shunts probably led to spontaneous resolution of hemolysis. Some authors point to the need for caution when using the ASO for occlusion of an LV pseudoaneurysm when the neck is greater than $20 \mathrm{~mm}$, as in the present case, with conventional surgery being the recommended approach in this group of patients.

\section{Expert opinion 3: Dr. Francesco Nappi}

The hemolysis that occurred is the effect of the loss of adhesion of the device to the ventricular wall, causing a leak.

As has been amply elucidated in the answer to the first question, the high mechanical stress of the left ventricle can favor the development of a loss of contact between the device and the ventricular wall. The smaller the leak, the larger the turbulence that is created when the blood flows out. For this reason, the lesion must be treated before it becomes too large to avoid the risk of hemolytic reaction.

\section{Question 3. What was the cause of the patient's death 2 months after the operation?}

\section{Expert opinion 1: Dr. Wilbert S. Aronow}

The cardiac, hepatic, and renal failure was responsible for the death of this patient.

\section{Expert opinion 2: Dr. Juan Lacalzada-Almeida}

In addition to the patient's irregular follow-up, the main cause of death could be the considerable LV free wall rupture, which resulted in the loss of a large quantity of healthy myocardium followed by an unavoidable decline in the pumping function of the heart. All of this could have caused death from heart failure and/or ventricular arrhythmia. Therefore, it would have been interesting to know the type of medical treatment on discharge, as well as the patient's degree of adherence to this treatment. Some authors have reported late reendothelialization and formation of thrombi in the closure device, as well as spontaneous formation of thrombi within the LV pseudoaneurysm owing to partial closure of the neck and slowing of blood flow in the interior, thus leading to a fatal cardioembolic cerebrovascular event as a possible complication of transcatheter closure. It would be necessary to analyze whether the patient was discharged with anticoagulant treatment or not, and, if he was, whether or not he took it appropriately.

\section{Expert opinion 3: Dr. Francesco Nappi}

There is a concrete probability that sudden death has occurred due to ventricular fibrillation in the presence of impaired ventricular function.

\section{Conclusions}

LVFWR is a rare but lethal complication of AMI. The 
effect of interventional therapy for LVFWR need to be taken seriously. The operation is limited because the lack of special interventional instruments. Future multicenter studies are required to identify patients best suited for interventional treatment timing. Additionally, further developments in devices and delivery techniques are required in order to optimize interventional outcomes.

\section{Acknowledgments}

Funding: None.

\section{Footnote}

Reporting Checklist: The authors have completed the CARE reporting checklist. Available at http://dx.doi.org/10.21037/ atm-20-6335

Conflicts of Interest: All authors have completed the ICMJE uniform disclosure form (available at http://dx.doi. org/10.21037/atm-20-6335). The authors have no conflicts of interest to declare.

Ethical Statement: The authors are accountable for all aspects of the work in ensuring that questions related to the accuracy or integrity of any part of the work are appropriately investigated and resolved. All procedures performed in studies involving human participants were in accordance with the ethical standards of the institutional and national research committees, and with the Helsinki Declaration (as revised in 2013). Written informed consent was obtained from the patient for publication of this manuscript and any accompanying images.

Open Access Statement: This is an Open Access article distributed in accordance with the Creative Commons Attribution-NonCommercial-NoDerivs 4.0 International License (CC BY-NC-ND 4.0), which permits the noncommercial replication and distribution of the article with the strict proviso that no changes or edits are made and the original work is properly cited (including links to both the formal publication through the relevant DOI and the license). See: https://creativecommons.org/licenses/by-nc-nd/4.0/.

\section{References}

1. Vatan B, Akdemir R, Cakar M, et al. Immediate primary transcatheter closure of left ventricular free wall rupture after myocardial infarction. Cardiology 2013;125:82-5.

2. Tsai IC, Lin MC, Jan SL, et al. Multidetector row computed tomography for percutaneous closure of a left ventricular free wall rupture after myocardial infraction. JACC Cardiovasc Interv 2015;8:e175-6.

3. Yudi MB, Love B, Nadir A, et al. Percutaneous closure of left ventricular pseudoaneursym with septal occluder device and coils: a multimodality imaging approach. JACC Cardiovasc Interv 2017;10:e159-61.

4. Acharya D, Nagaraj H, Misra VK. Transcatheter closure of left ventricular pseudoaneurysm. J Invasive Cardiol 2012;24:E111-4.

5. Omar S, Morgan GL, Panchal HB, et al. Management of post-myocardial infarction ventricular septal defects: a critical assessment. J Interv Cardiol 2018;31:939-48.

6. Harrison W, Ruygrok PN, Greaves S, et al. Percutaneous closure of left ventricular free wall rupture with associated false aneurysm to prevent cardioembolic stroke. Heart Lung Circ 2008;17:250-3.

7. Narayan RL, Vaishnava P, Goldman ME, et al. Percutaneous closure of left ventricular pseudoaneurysm. Ann Thorac Surg 2012;94:e123-5.

8. Bortnick AE, Gordon E, Gutsche J, et al. Percutaneous closure of a left ventricular pseudoaneurysm after Sapien XT transapical transcatheter aortic valve replacement. JACC Cardiovasc Interv 2012;5:e37-8.

9. Okamura H, Kimura N, Mieno M, et al. Sutureless repair for postinfarction left ventricular free wall rupture. J Thorac Cardiovasc Surg 2019;158:771-7.

10. Nappi F, Nenna A, Spadaccio C, et al. Predictive factors of long-term results following valve repair in ischemic mitral valve prolapse. Int J Cardiol 2016;204:218-28.

11. Tanaka K, Sato N, Yasutake M, et al. Clinicopathological characteristics of 10 patients with rupture of both ventricular free wall and septum (double rupture) after acute myocardial infarction. J Nippon Med Sch 2003;70:21-7.

12. Pineda-De Paz DO, Hernández-del Rio JE, GonzálezPadilla C, et al. Left ventricular free-wall rupture, a potentially lethal mechanical complication of acute myocardial infarction: an unusual and illustrative case report. BMC Cardiovasc Disord 2019;19:80.

13. Roberts WC, Burks KH, Ko JM, et al. Commonalities of cardiac rupture (left ventricular free wall or ventricular septum or papillary muscle) during acute myocardial infarction secondary to atherosclerotic coronary artery disease. Am J Cardiol 2015;115:125-40.

14. Jouan J, Tapia M, Cook RC, et al. Ischemic mitral valve 
prolapses: mechanisms and implications for valve repair. Eur J Cardiothorac Surg 2004;26:1112-7.

15. Kalra K, Wang Q, McIver BV, et al. Temporal changes in interpapillary muscle dynamics as an active indicator of mitral valve and left ventricular interaction in ischemic mitral regurgitation. J Am Coll Cardiol 2014;64:1867-79.

16. Sanz J, Weinsaft JW. Ischemic mitral regurgitation: is mitral valve physiology moving from global to local? J Am Coll Cardiol 2014;64:1880-2.

17. Arnaoutakis GJ, Zhao Y, George TJ, et al. Surgical repair of ventricular septal defect after myocardial infarction: outcomes from the Society of Thoracic Surgeons National Database. Ann Thorac Surg 2012;94:436-43; discussion $443-4$.

18. Ibanez B, James S, Agewall S, et al. 2017 ESC Guidelines for the management of acute myocardial infarction in patients presenting with ST-segment elevation: the Task Force for the management of acute myocardial infarction in patients presenting with ST-segment elevation of the European Society of Cardiology (ESC). Eur Heart J

Cite this article as: Zhong W, Liu Z, Fan W, Ou B, Zhong M, Zeng Z, Wang X, Aronow WS, Nappi F, Lacalzada-Almeida J, Zhong Z. Transcatheter closure for the treatment of pseudoventricular aneurysm after acute myocardial infarction: a case report. Ann Transl Med 2020;8(22):1528. doi: 10.21037/atm20-6335
2018;39:119-77.

19. Chamandi C, Abi-Akar R, Rodés-Cabau J, et al. Transcarotid compared with other alternative access routes for transcatheter aortic valve replacement. Circ Cardiovasc Interv 2018;11:e006388.

20. Kirker EB, Hodson RW, Spinelli KJ, et al. The carotid artery as a preferred alternative access route for transcatheter aortic valve replacement. Ann Thorac Surg 2017;104:621-9.

21. Wee IJY, Stonier T, Harrison M, et al. Transcarotid transcatheter aortic valve implantation: a systematic review. J Cardiol 2018;71:525-33.

22. Nishimura RA, Otto CM, Bonow RO, et al. 2017 AHA/ ACC focused update of the 2014 AHA/ACC guideline for the management of patients with valvular heart disease: a report of the American College of Cardiology/American Heart Association Task Force on Clinical Practice Guidelines. J Am Coll Cardiol 2017;70:252-89.

23. Chadwick RS. Mechanics of the left ventricle. Biophys J 1982;39:279-88. 